\title{
Delirium: Delay in Psychiatry Liaison Referral in a General Hospital - Case Study
}

Mariana Lázaro ${ }^{1}$, Luísa Gil', Amélia Lérias²

${ }^{1}$ Centro Hospitalar Psiquiátrico de Lisboa; ${ }^{2}$ Centro Hospitalar Lisboa Central - Hospital de São José

\section{Objectives}

Characterization of elderly inpatients diagnosed with Delirium;

Delirium subtype as a potencial factor associated with delay in psychiatry referral.

\section{Backgroung and Aims}

Delirium is extremely common in hospitalized older adults.

The first two key features involve disturbances in attention and cognition (memory, disorientation, language), and patients may experience a number of other disturbing symptoms (hallucinations, delusions, agitation).

Why is so important identifying and treating Delirium in an early phase?

Older patients with Delirium are more likely than other populations to develop hospital-acquired infections, pressure ulcers, and immobility and nutritional issues, as well as to have increased health care costs, longer hospital stays, and longterm care following discharge.

Can Delirium motor subtypes may play an important role in the diagnose and Delirium intervention?

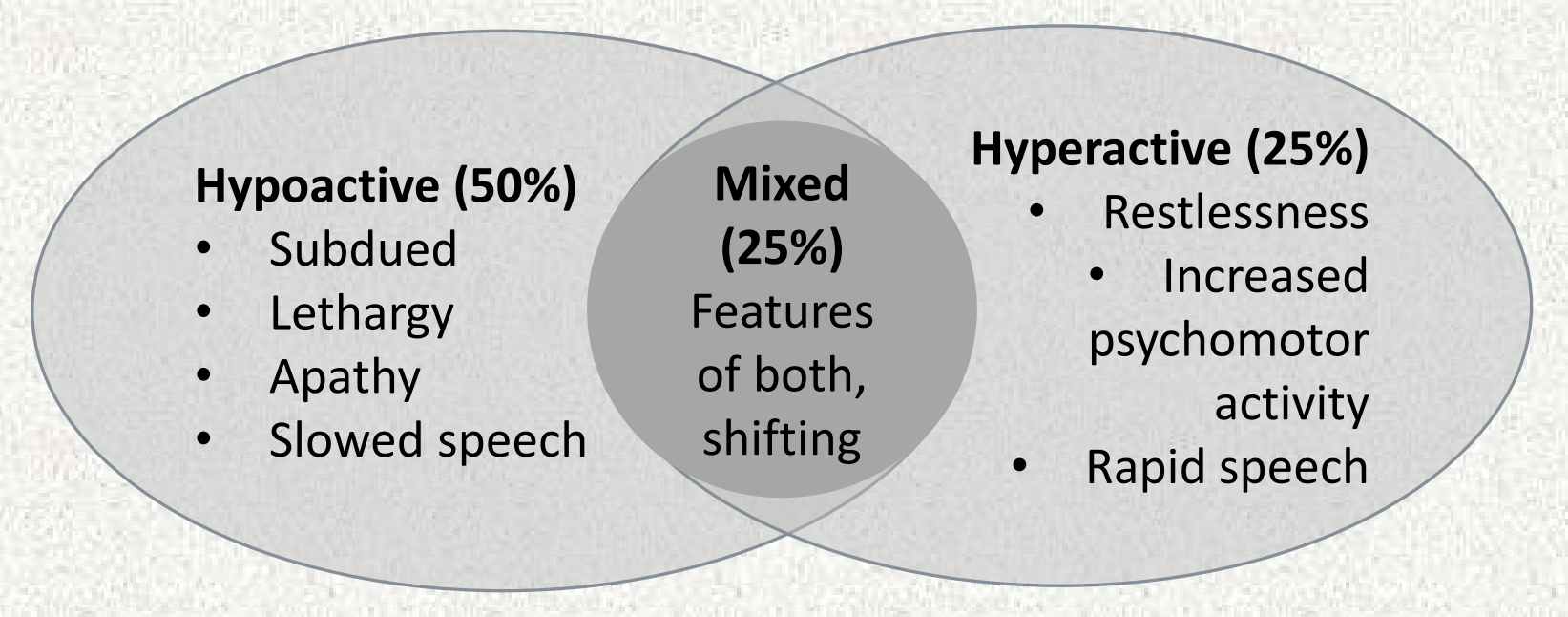

Hypoactive Delirium is

associated with a poorer

prognosis, potentially because

it is less frequently recognized
Hyperactive Delirium usually is easier to diagnose

\section{Material and Methods}

Observational-descriptive study of elderly patients (age $>65$ years old) admitted to Hospital de São José during the year 2017, assessed by Liaison Psychiatry ( $\mathrm{n}=\mathbf{2 3 6}$ patients).

Our convinience sample included 82 patients diagnosed with

Delirium. We evalueted:

- Motor subtype (hyperactive, hypoactive and mixed subtype);

- Mean duration of delay in psychiatry referral.

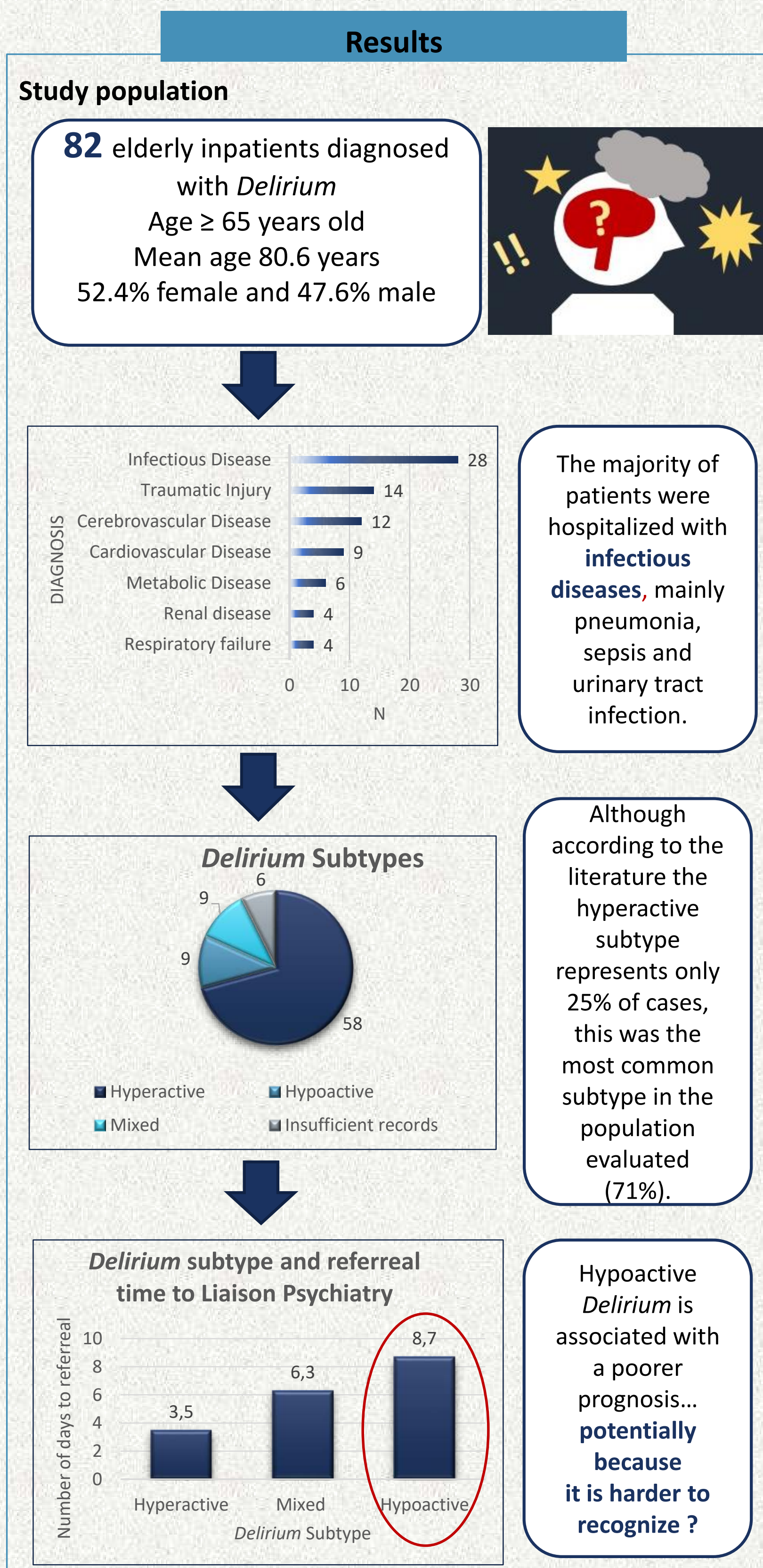

\section{Conclusion}

Even though there are several variables that should be considered, physicians may fail to identify patients with Delirium in the early phase, mainly hypoactive subtype, this may lead to underecognition and delay in therapeuthic intervention.

Efforts should be made to improve knowledge about Delirium early detection for a better patient outcome. 